\title{
Pseudodynamic testing and nonlinear substructuring of damaging structures under earthquake loading
}

\author{
A. Souid, A. Delaplace*, F. Ragueneau, R. Desmorat \\ LMT-Cachan (ENS Cachan/CNRS/Universite Paris 6/UniverSud Paris) \\ 61 avenue du Président Wilson, F-94230 Cachan, France
}

\begin{abstract}
Pseudodynamic or PSD testing with substructuring technique is a high relevant approach to capture the dynamic failure mechanisms of a structure. It allows to realistically test a structure subject to an earthquake with an accessible experimental equipment. We propose in this paper to apply such a PSD testing with substructuring to damaging structures, where the nonlinear behaviour is represented by an advanced damage model: an anisotropic damage model, initially proposed for monotonic applications, is upgraded in order to asses cyclic and seismic loading. The keypoint is the macroscopic representation of the microcracks closure (the cracks generated in tension close in compression) and its effect on stiffness recovery. The chain - PSD testing/advanced damage model/experimental measure setup based on image correlations - is successfully used for the study of the failure of a reinforced concrete frame structure.
\end{abstract}

Key words: Pseudodynamics, nonlinear substructuring, anisotropic damage, digital image correlation.

\section{Introduction}

Fine description of civil engineering structures failure under dynamic loading (impacts, earthquakes, explosions...) is still a high challenge, both from an

* Corresponding author: Tel.: +33 (0)147 402 238; fax: +33 (0)147 402240.

Email addresses: souid@lmt.ens-cachan.fr (A. Souid),

delaplac@lmt.ens-cachan.fr (A. Delaplace), raguenea@lmt.ens-cachan.fr

(F. Ragueneau), desmorat@lmt.ens-cachan.fr (R. Desmorat). 
experimental and a numerical points of view. The main difficulties are due to nonlinear behaviours of materials (concrete and steel) and of steel-concrete interfaces, and to the heterogeneous microstructure of concrete. The comprehension of the global failure can be approached by two ways: the experimental testing and the numerical modelling. Numerical simulations can be used to compute global structural failure. But robust and "universal" models are still needed to achieve such simulations, particularly for ultimate behaviour and preferably formulated for both uniaxial and multiaxial states of stress. On the other hand, laboratory experimental testing gives accurate information if realistic dimensions and pertinent boundary conditions are used. For civil engineering structures, the drawback is that heavy and expensive devices must be employed to perform the tests $[6,30,40]$.

However, it is not always necessary to make experimental tests on complete structures: often, failure of just a small part of the structure leads to global failure. Then, the so-called substructuring technique $[28,33,39]$, a combination between the numerical modelling (into which one can introduce the suitable elastic nonlinear or damage model of material behaviour) and an experimental test on a subpart of the structure, can be achieved to better understand the complete structure response. Furthermore, it is possible to numerically determine the inertia forces for performing static tests instead of dynamic ones: this simplification leads to pseudodynamic (or PSD) tests [24,38]. The main advantage of the coupled approach (PSD testing with substructuring) is that an ordinary experimental equipment is sufficient for testing instead of, for example, an expensive large-size shaking table. Then, many laboratories become able to test structures with realistic boundary conditions. Different improvements can be introduced to reinforce the reliability of the approach, from numerical to experimental aspects. We propose in this paper to focus on two enhancements: the consideration of an advanced nonlinear model for the computed substructure (an anisotropic damage model with microcracks closure effect), and the measurement setup (with digital image correlation).

Using a nonlinear modelling for the computed substructure is not always necessary: the substructuring technique is then based on the consideration that just a small part of the structure (the tested part) leads to the total failure, as the other part (the computed part) remains elastic or suffers a low-level damage (therefore neglected). However, damage $D$, even low, changes the natural frequencies of the complete structure as the Young modulus (and therefore the stiffness) is affected by damage as $\tilde{E}=E(1-D)$. The frequencies change should be accounted for, for instance by using a nonlinear model for the computed substructure: we propose next the use of an anisotropic damage model. For the substructuring technique, one has to perform efficient numerical computations to reduce the waiting time of the experimental part. Note that the last feature becomes an obligation for real time testing [10]. This goal is reached by considering the lowest possible number of degrees of freedom (dof) 
and a robust model. We propose in this paper to use an intermediate structural discretization by using the multifiber theory $[2,13,36]$ to reduce the total number of dof, coupled as a challenge with an anisotropic damage model. The choice concerning the model is governed by the fact that:

- one wants to use a realistic model for concrete, material exhibiting oriented microcracking state during loading.

- including more physics (damage anisotropy) can simplify the model and reduce the number of material parameters (five in the present case, including elastic parameters).

- one wants to propose and to use a 3D damage model suitable for 3D application, keeping then the possibility of Finite Element 3D reanalysis of the tested structure.

- considering a first damage variable for tension and a second damage variable for compression is not thermodynamically acceptable. A state damage variable represents the state of microcracking of the material and it cannot be defined by reference to the loading sign. Damage loading induced anisotropy corresponds to the physical observations.

Experiment measurement for PSD testing with substructuring is an important concern. We propose to use digital image correlation techniques to perform measurements during testing $[37,18]$. In this way, the recorded data are no more limited to localised measurements. This tool increases the interest of the approach by providing the displacement and strain fields of the tested substructure, a fine description of cracking pattern (length, opening) and an exact characterisation of the boundary conditions. All these observations can be advantageously used for a further validation of a 3D model.

The present work describes different results of pseudo-dynamic tests obtained with substructuring technique, using then both an efficient numerical model and digital image correlation techniques. The tests are conducted for a two bays and two levels reinforced concrete frame which is subjected to a twocomponent earthquake. As mentioned earlier, the test is performed on an ordinary experiment setup. The basis of the substructuring technique and of the pseudodynamic test is recalled first. The anisotropic damage model is introduced and implemented within a simplified multifiber formulation. Lastly, the experimental setup is described and results obtained with three different reinforced concrete beams are given. 


\section{Pseudodynamic testing and substructuring technique}

\subsection{Pseudodynamic testing}

Evaluation of the earthquake response of a structural system is usually performed using a shaking table. However, experiments for large scale structures are difficult to perform, generally due to table capacity limitations $[19,40]$. An alternative way for testing full or large scale structures is the PsD testing $[24,38]$, an experimental technique developed to evaluate the earthquake performance of structure samples in a laboratory by means of computer-controlled simulation. It is a hybrid method, in which the structural displacements resulting from the earthquake-induced inertia forces are computed by using a stepwise integration procedure and applied quasi-statically to the tested specimen. The resulting resistance forces are measured and fed back to the computational model as part of the input for the next calculation step. The dynamic equilibrium equations including material nonlinearities to be solved in a finite element framework can be expressed by:

$$
\mathbf{M} a_{n+1}+\mathbf{C} v_{n+1}+r_{n+1}\left(u_{n+1}\right)=f_{n+1}
$$

where $\mathbf{M}, \mathbf{C}$ are the mass and damping matrices, $a_{n+1}, v_{n+1}, u_{n+1}, r_{n+1}$ and $f_{n+1}$ are respectively the relative acceleration, velocity, displacement, restoring force and loading vectors at time $t_{n+1}$. Knowing the variables at time $t_{n}$, one can compute the displacement and the velocity at time $t_{n+1}$ by using a numerical time integration scheme [31,27], for example the Newmark implicit one:

$$
\begin{aligned}
& u_{n+1}=\tilde{u}_{n+1}+\beta(\Delta t)^{2} a_{n+1} \\
& \tilde{u}_{n+1}=u_{n}+\Delta t v_{n}+\frac{(\Delta t)^{2}}{2}(1-2 \beta) a_{n} \\
& v_{n+1}=v_{n}+(1-\gamma) \Delta t a_{n}+\gamma \Delta t a_{n+1}
\end{aligned}
$$

where $\beta$ and $\gamma$ are the Newmark parameters. The acceleration $a_{n+1}$ can be computed from (1) if the restoring force $r_{n+1}\left(u_{n+1}\right)$ is known: these forces are directly measured and fed back to the computational model as part of the input. We will consider in this study the $\alpha$-OS scheme $[15,8,28,34]$. This later is based on the modification of the equation of motion through the introduction of a numerical dissipation factor $\alpha$

$$
\begin{aligned}
& \mathbf{M} a_{n+1}+(1+\alpha) \mathbf{C} v_{n+1}-\alpha \mathbf{C} v_{n}+(1+\alpha) r_{n+1}-\alpha r_{n}= \\
& (1+\alpha) f_{n+1}-\alpha f_{n}
\end{aligned}
$$


and through the splitting of the stiffness force vector into a measured part and a linearized one:

$$
r_{n+1}\left(u_{n+1}\right) \cong \tilde{r}_{n+1}\left(\tilde{u}_{n+1}\right)-\mathbf{K}^{I} \tilde{u}_{n+1}
$$

where $\mathbf{K}^{I}$ is a stiffness matrix which is often taken as the elastic one. The corresponding Newmark's parameters are $\beta=(1-\alpha)^{2} / 4$ and $\gamma=1 / 2-\alpha$. If necessary, tests can be ran on expanded time scale $[24,40]$ and then performed at low loading rate. This method can use conventional inexpensive quasi-static equipment to realise dynamic testing. The main feature is that all inertia components are obtained numerically, and no additional masses have to be considered even for small scale experiment. It makes full scale testing feasible, at least as long as a sufficient strong floor and reaction walls are available. The drawback in such a method is that it is not adapted to massive structures, as dams, whose masses are distributed uniformly on the structural elements. One has to consider that masses can be condensed in the tested degrees of freedom.

\subsection{Substructuring technique}

The PsD testing with substructuring can significantly reduce the cost of the experimental tests conducted to capture the seismic behaviour of structures [6]. In substructuring technique, a physical model is built only for the part where severe nonlinearity is expected (the physical substructure), while the remaining parts are computed (the numerical substructure). This method initially developed by [24] and [38] has been considerably extended by researchers at Ispra JRC [3]. The numerical part is modelled by using a finite element code in a first computer connected through a network with the computers dedicated to the experimental procedures for the pseudodynamic test. The displacement at the interface between the physical (tested) and numerical (computed) substructures is obtained and applied to the tested specimen by hydraulic actuators (an example is given in Fig. 7). The resulting resistance forces are measured by load cells and fed back to the numerical model, together with the next increment of earthquake ground motion. A new interface displacement is then computed and applied to the tested specimen, and the loop is repeated until the test is completed $[5,40]$. This basic algorithm of partitioned procedures (called the staggered procedure [29]) is inherently sequential: the numerical substructure simulation procedure is in advance with respect to the tested one as illustrated in figure 1.

Many integration schemes (explicit, implicit, $\alpha$-OS method) can be applied to the procedures for PsD tests $[28,34]$. All of these schemes lead to the following equation to be solved for the entire structure: 
Simulated substructure

Tested substructure
$(1)$

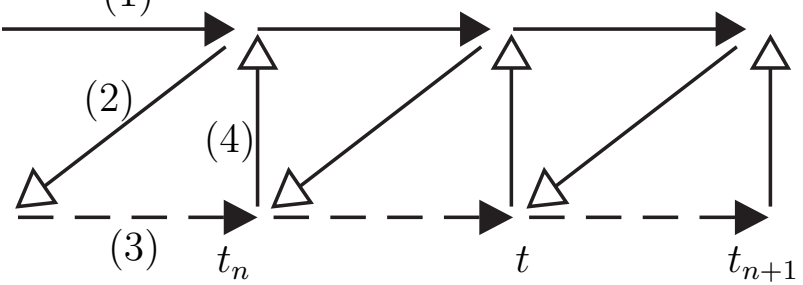

Fig. 1. The conventional staggered procedure [29].

$$
\hat{\mathbf{M}} a_{n+1}=\hat{f}_{n+1}
$$

where $\hat{\mathbf{M}}$ is the generalised mass matrix and $\hat{f}_{n+1}$ is the generalised force. If the $\alpha$-Operator Splitting scheme is used, these generalised terms become:

$$
\begin{aligned}
& \hat{\mathbf{M}}=\mathbf{M}+\gamma \Delta t(1+\alpha) \mathbf{C}+\beta \Delta t^{2}(1+\alpha) \mathbf{K}^{I} \\
& \hat{f}_{n+1}=(1+\alpha) f_{n+1}-\alpha f_{n}+\alpha \tilde{r}_{n}-(1+\alpha) \tilde{r}_{n+1} \\
& \quad+\alpha \mathbf{C} \tilde{v}_{n}-(1+\alpha) \mathbf{C} \tilde{v}_{n+1}+\alpha\left(\gamma \Delta t \mathbf{C}+\beta \Delta t^{2} \mathbf{K}^{I}\right) a_{n}
\end{aligned}
$$

By distinguishing the internal degrees of freedom of the computed substructure $(i, j)$, the external degrees of freedom of the tested substructure $(I, J)$ and the common interface degrees of freedom of the two substructures $(\theta, \delta)$, one can rewrite equation (7) as:

$$
\left[\begin{array}{ccc}
{ }^{S} \hat{\mathbf{M}}_{i j} & { }^{S} \hat{\mathbf{M}}_{i \theta} & 0 \\
{ }^{S} \hat{\mathbf{M}}_{\delta j}{ }^{S} \hat{\mathbf{M}}_{\delta \theta}+{ }^{T} \hat{\mathbf{M}}_{\delta \theta}{ }^{T} \hat{\mathbf{M}}_{\delta J} \\
0 & { }^{T} \hat{\mathbf{M}}_{I \theta} & { }^{T} \hat{\mathbf{M}}_{I J}
\end{array}\right]\left[\begin{array}{c}
a_{j}^{n+1} \\
a_{\theta}^{n+1} \\
a_{J}^{n+1}
\end{array}\right]=\left[\begin{array}{c}
{ }^{S} \hat{f}_{i}^{n+1} \\
{ }^{S} \hat{f}_{\delta}^{n+1}+{ }^{T} \hat{f}_{\delta}^{n+1} \\
{ }^{T} \hat{f}_{I}^{n+1}
\end{array}\right]
$$

where the superscripts $S$ and $T$ denote the $S$ imulated and Tested substructures. A static condensation applied to interface nodes allows to treat only two systems: the first one for the modelled substructure and the second one for the tested substructure. Note that the time discretization used for computing can be different from the time discretization used during testing but the exchanged data between the different procedures must be synchronous. Convergence, accuracy and experimental phenomena lead to the choice of numerical time integration scheme and the integration time step.

The restoring forces needed to compute $\hat{f}_{n+1}$ (equation 9 ) can be obtained experimentally from the tested substructures and numerically from the finite element simulation. Although the failure of just the tested part leads to the collapse of the global structure, and as already mentioned, a low level of damage can appear in the computed substructure. The main effect of this low damage growth is the classical decrease of the natural frequencies of the com- 
plete structure, and then a change in the global response. The corresponding nonlinear damage/multifiber modelling is described next.

\section{$3 \quad$ Nonlinear modelling at local and global scales}

An important aspect when using a nonlinear model for the simulated substructure is the robustness and the rapidity of the computation to avoid excessive waiting time for the tested substructure. When dealing with cyclic or seismic behaviour of concrete, one has in a general manner to account for:

(i) the decrease in material stiffness as the microcracks open,

(ii) the (partial) stiffness recovery as microcracks closure occurs,

(iii) the large dissymmetry tension/compression and induced anisotropy,

(iv) inelastic strains concomitant to damage.

Concerning the robustness of the model, the number and the physical meaning of the material parameters are important features to be considered, as the simplicity of the final constitutive equations and of numerical implementation. Except for the feature (iv), these different goals can be obtained with high efficiency by considering an anisotropic damage model [9], the keypoint being the consideration of a strain based damage criterion (Mazars criterion is used next). Moreover, a classical multifiber modelling for the discretization of the simulated substructure will be used to decrease even more the computation time.

\subsection{Anisotropic damage model for concrete and cyclic loading}

The proposed anisotropic damage model is built within the thermodynamic framework. Following [9], the Gibbs free enthalpy $\rho \psi^{*}$ is split into a hydrostatic part and a deviatoric one ( $\rho$ is the density):

$$
\begin{aligned}
\rho \psi^{*}=\frac{1+\nu}{2 E} \operatorname{Tr}\left[\underline{H} \underline{\sigma}_{+}^{D} \underline{H} \underline{\sigma}_{+}^{D}+\left\langle\underline{\sigma}^{D}\right\rangle_{-}\right. & \left.:\left\langle\underline{\sigma}^{D}\right\rangle_{-}\right] \\
& +\frac{1-2 \nu}{6 E}\left[\frac{\langle\operatorname{Tr} \underline{\sigma}\rangle_{+}^{2}}{1-\operatorname{Tr} \underline{D}}+\langle\operatorname{Tr} \underline{\sigma}\rangle_{-}^{2}\right]
\end{aligned}
$$

where the damage state is represented by the 2 nd order tensor $\underline{D}$, where $E$ and $\nu$ are the Young modulus and Poisson ratio of initially isotropic elasticity, $\underline{\sigma}^{D}=\underline{\sigma}-\frac{1}{3} \operatorname{Tr}[\underline{\sigma}] \underline{1}$ is the deviatoric stress, $\underline{H}=(\underline{1}-\underline{D})^{-1 / 2} \cdot \underline{\sigma}_{+}^{D}$ is a special positive part allowing to differentiate the state potential $[21,23]$ and $\left\langle\underline{\sigma}^{D}\right\rangle_{-}$is the negative part in terms of principal values of tensor $\underline{\sigma}^{D}$. Lastly, the elasticity 
law reads:

$$
\begin{aligned}
\underline{\varepsilon}=\rho \frac{\partial \psi^{*}}{\partial \underline{\sigma}}=\frac{1+\nu}{E} \operatorname{Tr}\left[\left(\underline{H} \underline{\sigma}_{+}^{D} \underline{H}\right)^{D}\right. & \left.+\left\langle\underline{\sigma}^{D}\right\rangle_{-}^{D}\right] \\
& +\frac{1-2 \nu}{3 E}\left[\frac{\langle\operatorname{Tr} \underline{\sigma}\rangle_{+}}{1-\operatorname{Tr} \underline{D}}+\langle\operatorname{Tr} \underline{\sigma}\rangle_{-}\right] \underline{1}
\end{aligned}
$$

with positive (respectively negative) part of a scalar $x$ being $\langle x\rangle_{+}=\max (0, x)$ (respectively $\langle x\rangle_{-}=\min (0, x)$ ) As for plasticity, the elasticity domain can be defined through a criterion function $f$ such as the domain $f<0$ corresponds to elastic loading or unloading. Many criteria can be used, written in terms of stresses such as plasticity criteria, strains, or strain energy release rate density leading or not to dilatancy in compression. The purpose here is to build a constitutive model with a restricted number of material parameters suitable for seismic loading, robust and easy to implement in a finite element code. Dilatancy will not be taken into account and one will accept an open criterion for the triaxial compressive states. These remarks lead us to the simple choice of Mazars criterion [25], function of the positive strains $\left\langle\varepsilon_{I}\right\rangle_{+}$, positive parts of the $I$ th principal strains $\varepsilon_{I}$,

$$
f=\hat{\varepsilon}-\kappa, \quad \text { with } \quad \hat{\varepsilon}=\sqrt{\sum\left\langle\varepsilon_{I}\right\rangle_{+}^{2}}
$$

where $\hat{\varepsilon}$ is the equivalent strain for quasi-brittle materials. The initial value $\kappa_{0}$ of $\kappa$-function is the elastic strain limit in tension. The damage anisotropy is assumed governed by the positive strains as the tensorial expression

$$
\underline{D}=\dot{\lambda}\langle\underline{\varepsilon}\rangle_{+}
$$

where $\dot{\lambda}$ is the damage multiplier determined from the consistency condition $f=0, \dot{f}=0$. Concerning the consolidation function $\kappa$, it accounts for damage increase in tension as well as in compression, but also for microcracks closure from tension to compression, with an adaptative choice for its argument, next the effective damage $d_{\varepsilon}$ such as:

$$
\kappa=\kappa\left(d_{\varepsilon}\right) \quad \text { with: } d_{\varepsilon}=\frac{\underline{D}:\langle\underline{\varepsilon}\rangle_{+}}{\max \left(\varepsilon_{I}\right)}
$$

Then, $d_{\varepsilon}=D_{1}$ in tension parallel to $x$-axis (whatever the amount of the damage generated in compression $D_{2}$ ) and $d_{\varepsilon}=2 D_{2}$ in compression (whatever the amount of the damage generated in tension $D_{1}$ ) representing then damage deactivation, i.e. microcracks closure. The choice (15) recovers the choice $d_{\varepsilon}=$ $\operatorname{Tr} \underline{D}$ made in previous monotonic modelling. The function $\kappa$ is chosen as in $[9]$ : 


$$
\kappa\left(d_{\varepsilon}\right)=a \cdot \tan \left[\frac{d_{\varepsilon}}{a A}+\operatorname{atan}\left(\frac{\kappa_{0}}{a}\right)\right]
$$

The numerical implementation of the above anisotropic damage model is quite simple in uniaxial stresses case and is easily applicable for multifiber modelling. Figure 2 shows the response of the model in the case of a damaging tension followed by compression. The microcracks due to tension close in compression. The material parameters used are: $E=25 \mathrm{GPa}, \nu=0.2, \kappa_{0}=7 \times 10^{-5}, A=$ $5200, a=2.97 \times 10^{-4}$ and will be used for the experimental study. A perfect plasticity model will be used to represent the reinforcing steels behaviour, with a Young's modulus of $210 \mathrm{GPa}$ and a yield stress of $500 \mathrm{MPa}$.

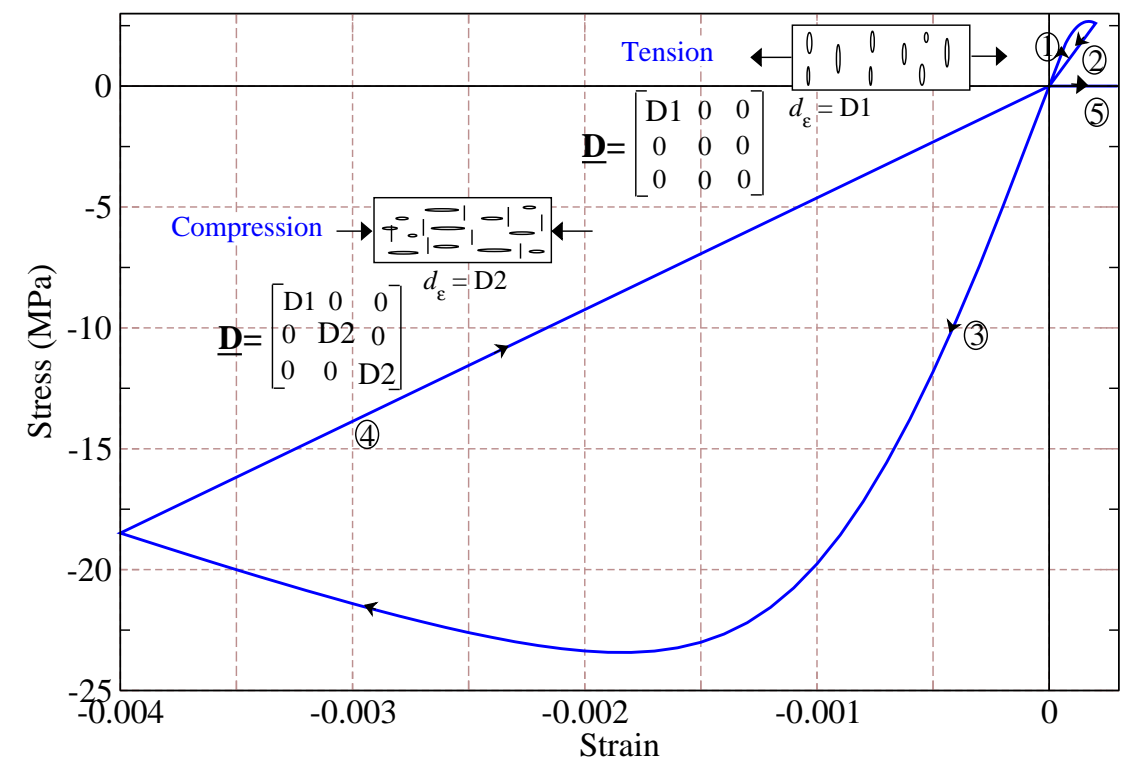

Fig. 2. Stress-strain response for concrete (loading in direction 1)

\subsection{Multifiber structural modelling}

Finite element numerical simulations are useful to describe the complex behaviour of structural elements subject to 3D coupled loadings. For a simple reason of excessive computational costs, complete 3D approaches are usually avoided in structural dynamics of civil engineering structures. So, for large scale computations of structures, pragmatism at global level has to be targeted if some physical local phenomena have to be described. An intermediate structural discretization (between complete 3D approach and macro-element method) makes use of the multifiber theory $[2,13,36]$. It allows for simple, robust and efficient computations at the global level by introducing a low number of degrees of freedom insuring global convergence in the fastest way. The classical Timoshenko beam model is considered for the structural components of a complex civil engineering structure $[2,36]$. The refined constitutive 


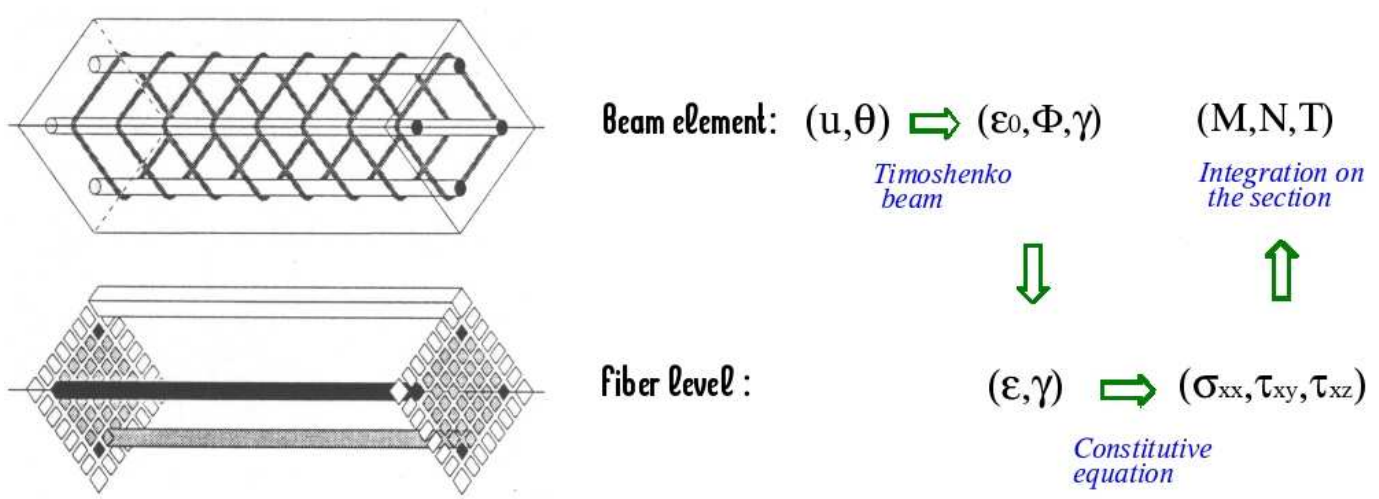

Fig. 3. Multifiber beam for concrete structures (after Guedes et al. 1994)

equations are kept unchanged at the local level, but due to the beam kinematics, those are implemented using only their uniaxial feature. This later guarantees efficient and easy stress integration on the section.

The kinematics hypothesis assumes no distortion or warping of a cross section (if needed see [26]). For a multifiber beam model, the nonlinear behaviour implies coupling between axial force $N$ and bending moment $M$. It is thus necessary that the axial force and bending moment have the same variation along the element. The latter is not satisfied for an Euler-Bernoulli multifiber beam element, where axial strain remains constant along the element whereas the bending strain is linear. This problem may become important in the nonlinear range where a linear curvature along the beam implies a linear variation of the axial strain due to the shift of the neutral axis. So as to remove this incompatibility of variations for axial strain and curvature one can use an enriched description of the axial strain field for the Euler-Bernoulli type finite element or one can lower the interpolation of the curvature to accommodate the axial strain variation. The latter choice is retained allowing to deal with Timoshenko beam finite element. Knowing that the Timoshenko beam theory assumes that the plane sections remain plane after deformation but not necessarily normal to the beam axis. The section stiffness matrix $\mathbf{K}$ is calculated in elasticity as given by [13]. The general scheme (see figure 3) consists in computing the local strains in each fiber from the nodal displacements and rotations through the Timoshenko's beams equations. Note that even if the simple 1D multifiber theory is used, the coupling with the anisotropic damage model allows to distinguish cracks due to tension (perpendicular to the beam axis) from cracks due to compression (parallel to the beam axis). 


\section{Experimental PsD tests on reinforced concrete substructures}

\subsection{Description of the structure}

The substructuring technique is applied in pseudodynamics to the study of a reinforced concrete structure, shown in figure 4. A distributed loading mass ( $m=0.75$ tons $/ \mathrm{m})$ is applied on the upper beam and on the right middle one. A concentrated loading mass ( $M=2.25$ tons) is applied in the middle of the left beam P1P2. In that way, the failure of the last is expected to cause the collapse of the structure, leading to the substructuring decomposition shown in figure 5. In order to limit the experimental setup to one actuator, additional conditions are assumed (not fully representative of a real structure):

- displacements of the beam ends P1P2 are assumed equal,

- vertical and horizontal associated reacting forces are assumed equal,

- the mass $M$ is equally distributed on P1 and P2 for the computed substructure,

- the horizontal and vertical seismic excitations have been calibrated in order to impose a strong damage on the tested beam, and to impose a low level of damage in the computed substructure.

These assumptions nevertheless allow us to perform the one-actuator testing and to demonstrate the feasibility of the proposed analysis, from nonlinear PSD testing with substructuring to digital image correlation and failure modes observation.

are obviously not representative of a real structure, and one has to consider realistic boundary conditions adapted to the studied structure.

All the frame elements, excepted the beam P1P2, have a $15 \times 15 \mathrm{~cm}^{2}$ section (of type A) and are longitudinally reinforced by four steel bars of diameter $10 \mathrm{~mm}$. The beam P1P2 has a 20 (height) $\times 15$ (wide) $\mathrm{cm}^{2}$ section (of type $\mathrm{B}$ ), and three different reinforcements R1, R2 and R3 are considered (table 1): $\mathrm{R} 1$ has a normal reinforcement, R2 has overspacing stirrups, and R3 has a weak longitudinal reinforcement. The clamped frame is loaded with a dynamic seismic signal (figure 6) applied on its foundation. Two synthetic signals are applied, one in horizontal direction based on the code PS92 [1], one in vertical direction with its bandwidth centred on $14-24 \mathrm{~Hz}$ frequencies in order to excite the vertical mode of the structure (Fig. 6). 


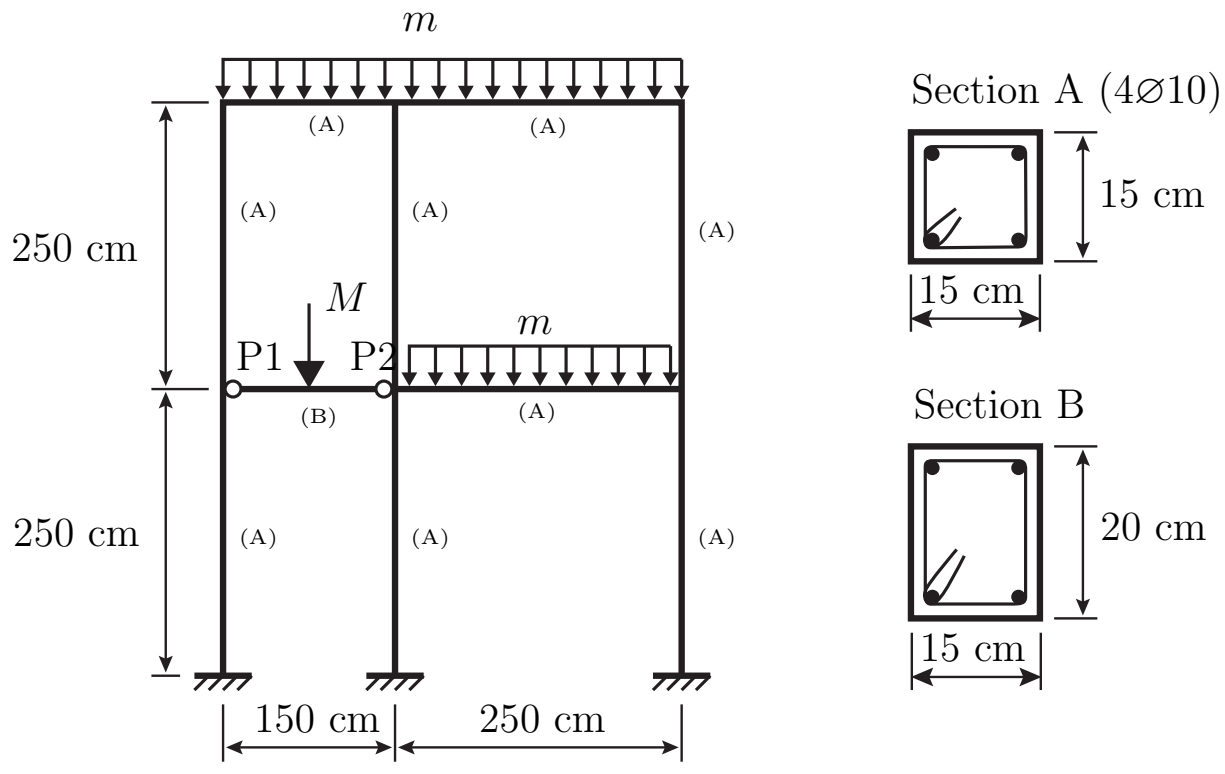

Fig. 4. The structure geometry.
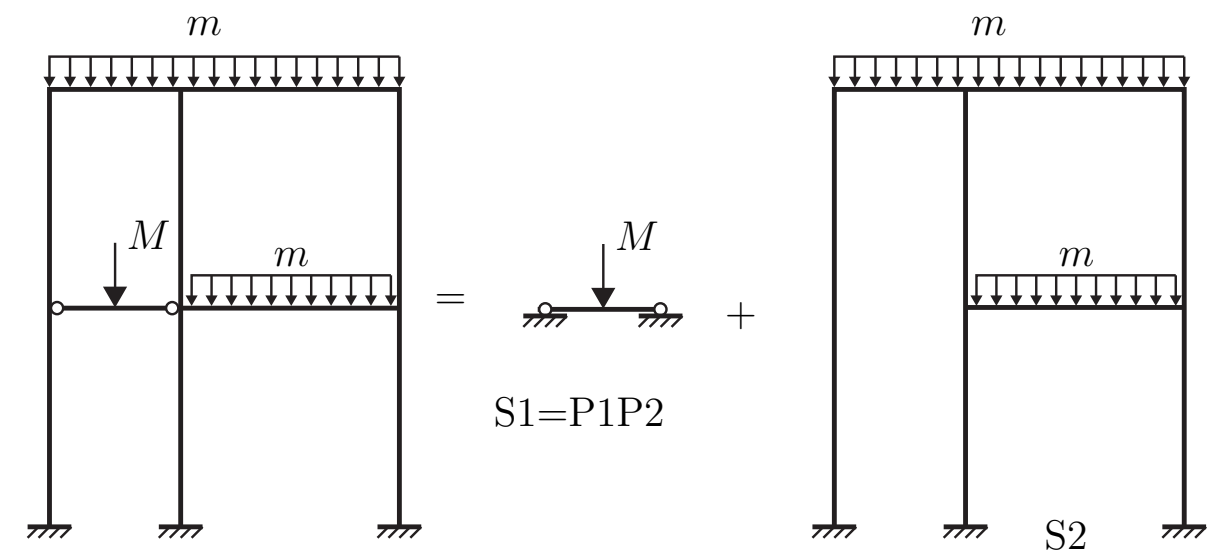

Fig. 5. Decomposition of the structure into tested substructure S1 and simulated substructure S2.

\begin{tabular}{|l|c|c|}
\hline & $\begin{array}{c}\text { Longitudinal reinforcement } \\
(\mathrm{mm})\end{array}$ & $\begin{array}{c}\text { Transversal reinforcement } \\
(\text { diameter }(\mathrm{mm}) / \mathrm{spacing}(\mathrm{cm}))\end{array}$ \\
\hline reinforcement 1 (R1) & $4 \varnothing 10$ & $\varnothing 6 / 15 \mathrm{~cm}$ \\
\hline reinforcement 2 (R2) & $4 \varnothing 12$ & $\varnothing 6 / 30 \mathrm{~cm}$ \\
\hline reinforcement 3 (R3) & $4 \varnothing 8$ & $\varnothing 6 / 15 \mathrm{~cm}$ \\
\hline
\end{tabular}

Table 1

The three different reinforcements used for the beam P1P2.

4.2 Experimental setup

The strength of a substructuring analysis is that just a part (the presumed critical one) of the whole structure has to be experimentally tested. In the 

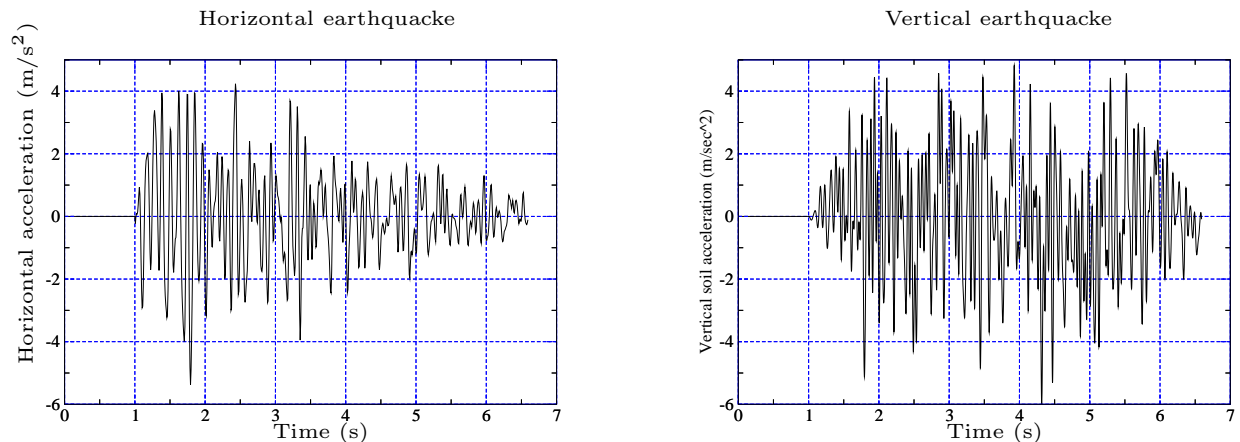

Fig. 6. Horizontal (left) and vertical (right) earthquake components applied on the foundation.
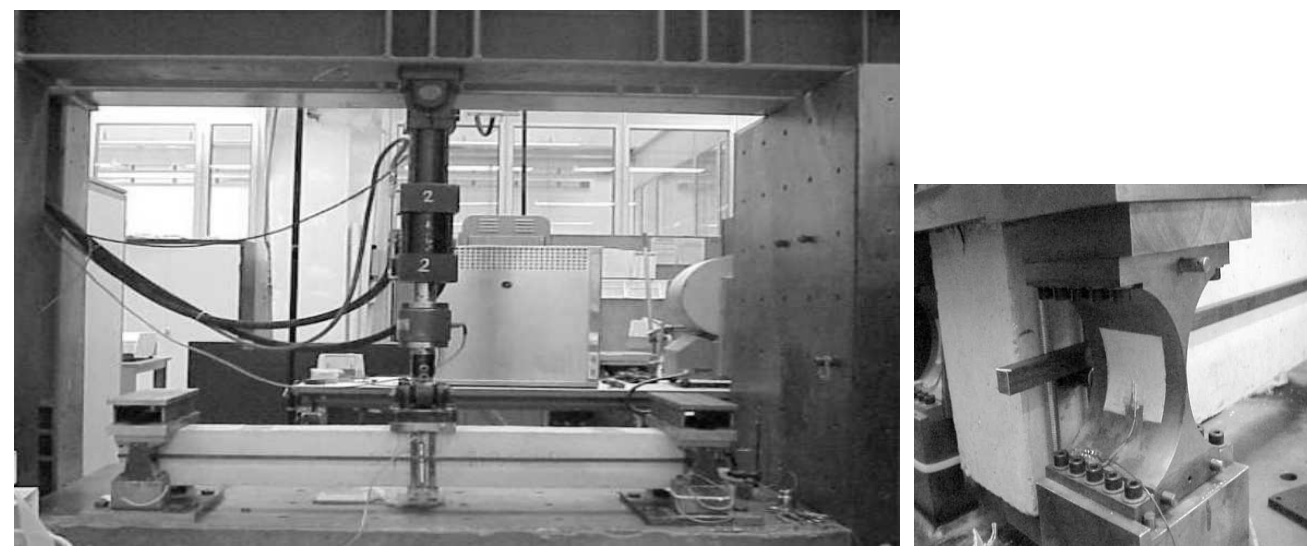

Fig. 7. Experimental setup (left) and hinge device (right).

present case, substructure S1 is tested with a simple three-point bend setup. The setup has perfect hinges at the beam extremities (Fig. 7), that guarantees quasi-perfect boundary conditions [20].

An external linear variable differential transformer (LVDT) $(0-50 \mathrm{~mm})$ is used to measure the applied displacement. The control of the vertical hydraulic actuator is made by comparing the applied deflection to measurements done by this external LVDT. The piloting range used is $\pm 50 \mathrm{~mm}$ and corresponds to \pm 10 volts. The stiffness $\mathbf{K}^{I}$ which has been introduced in equation (6) for $\alpha-O S$ scheme is taken as the experimental measured stiffness at the end of the static loading. The equation of motion of the tested substructure is solved in a Personal Computer by PSEUDYN program (done in Labview and developed at LMT-Cachan). Labview is a graphical language program whose virtual instruments, named VI, can easily ensure calculations, control and acquisition measurements. Moreover, these instruments can perform the communication procedures with another Personal Computer on the network used to simulate the numerical response of the studied substructure S2. In fact, PSEUDYN controls the hydraulic actuator with a Teststar ${ }^{\mathrm{MTS}}$ program in an external mode. 
The computation of the response of the substructure S2 is performed in the CEA finite element code Cast3M [7]. A viscous damping is considered for this substructure by using a Rayleigh damping: $2 \%$ for the first eigen mode and $1 \%$ for the second one are dissipated as $\mathbf{C}=0.25 \mathbf{M}+0.0004 \mathbf{K}^{I}$. The time step of the loading is taken as $\Delta t=1.46 \times 10^{-3} \mathrm{~s}$.

Digital image correlation is obtained by using a Canon EOS 350D camera. The studied zone of an image is named the Region Of Interest (ROI). Figure 8 shows a typical dimension of a ROI and the corresponding resolution. The width of this zone is $458 \mathrm{~mm}$. The image resolution is $1737 / 458=3.79$ pixels $/ \mathrm{mm}$. The system used to apply the alternate displacement response loading is shown also in figure 8. We consider here the ROI as the entire photographed zone to be analysed by the Digital Image Correlation technique (DIC). Thanks to this technique of correlation, it is possible to characterise in a very precise way displacements and deformations starting from a reference digital image $[17,18,14]$. One obtains the displacements fields of the ROI. Therefore, the cracks opening is known at the moment the image is taken. In other words, one can determine the cracking states of the studied zones of the structure at any moment of the seismic response. The images correlation is done by CORRELI $^{\mathrm{LMT}}$ software [16] which is a LMT-Cachan data-processing application dedicated to field measurements. From a reference digital image and a deformed digital image, the software determines the plane field of displacements and deformations which exists between two successive images.

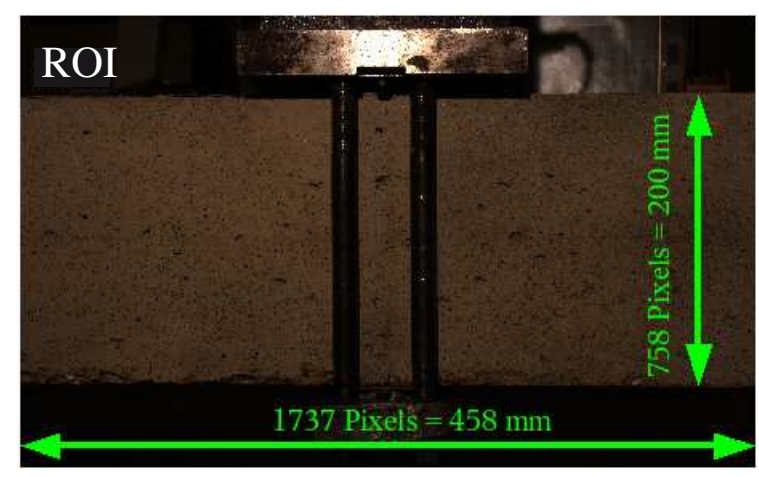

Fig. 8. An example of a Region Of Interest (ROI) and the corresponding resolution.

\subsection{Results}

The reinforcements of the beams are expected to reveal three failure mechanisms. For each reinforcement type, two beams have been tested. Figure 9 shows the applied displacements for the three reinforcements with respect to the time (left diagrams) and the evolution of the measured force versus the applied displacement (right). 
- Reinforcement R1 corresponds to a ductile failure with hardening of the steel bars, the beam keeping some rigidity at the end of the loading. The damping is clearly visible with this reinforcement and one can use this result to calibrate internal damping under cyclic loading.

- Reinforcement R2, with overspacing stirrups, fails suddenly due to shear loading after 3.6 or $3.8 \mathrm{~s}$ of loading.

- Reinforcement R3, with under-reinforced longitudinal bars, fails suddenly after the breaking of the longitudinal bars after 3.1 or $3.3 \mathrm{~s}$ of loading.
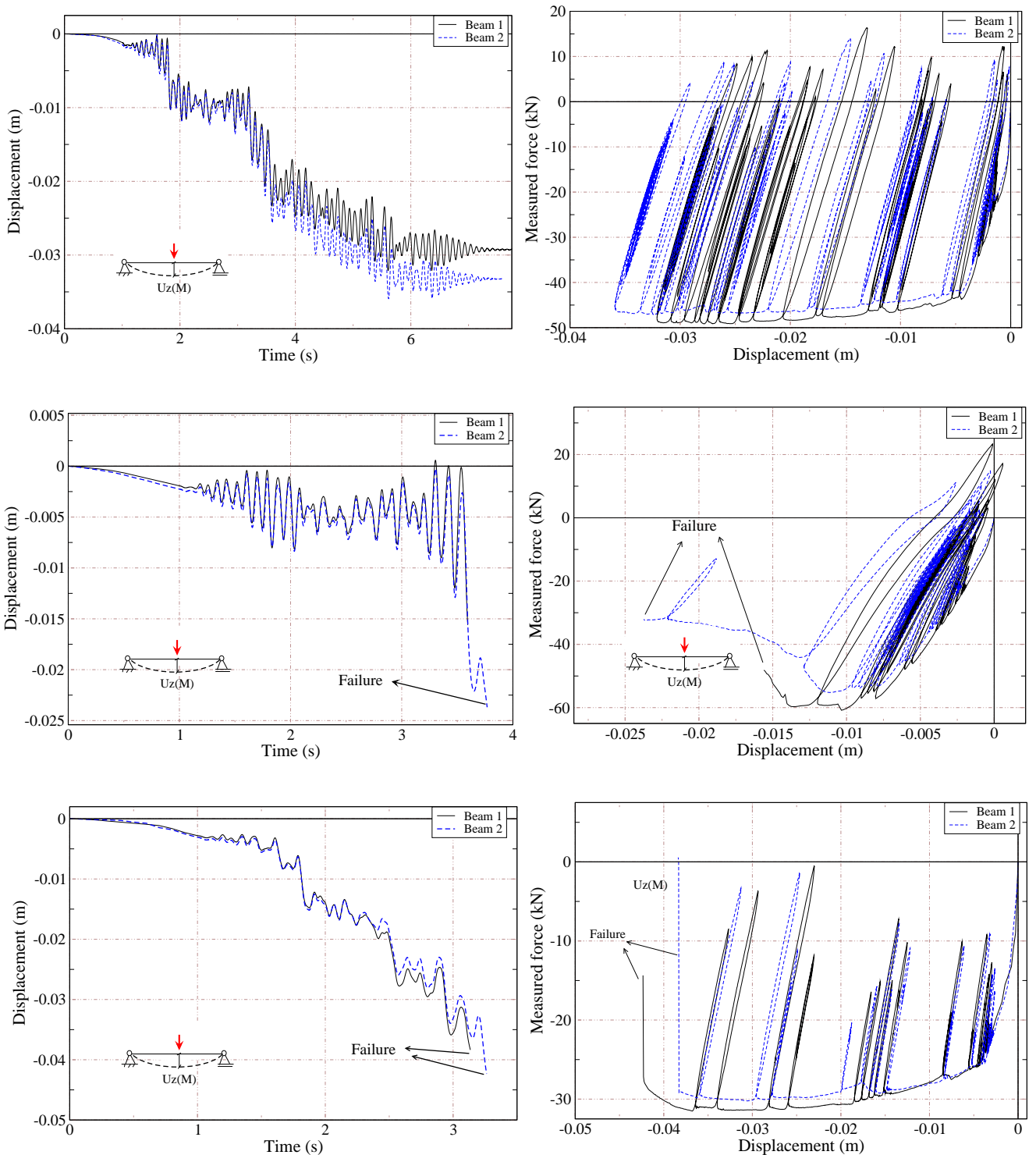

Fig. 9. Applied displacement versus time (left) and measured force versus applied displacement (right) for the three different reinforcements (top: R1, middle: R2, bottom: R3).

The cracking pattern obtained for the first two reinforcements are presented in figure 10 and 11 . The cracks are clearly visible on both the strain and displacement fields. 
- Failure of reinforcement R1 beam shows mainly cracks perpendicular to the beam axis due to alternate tension of the top and bottom faces of the beam.

- Failure of reinforcement R2 reveals cracks typical of shear mode. The crack opening is gained from the displacement fields.

Unfortunately, brittle failure of R3 beams doesn't give a reliable measure with Digital Image Correlation.
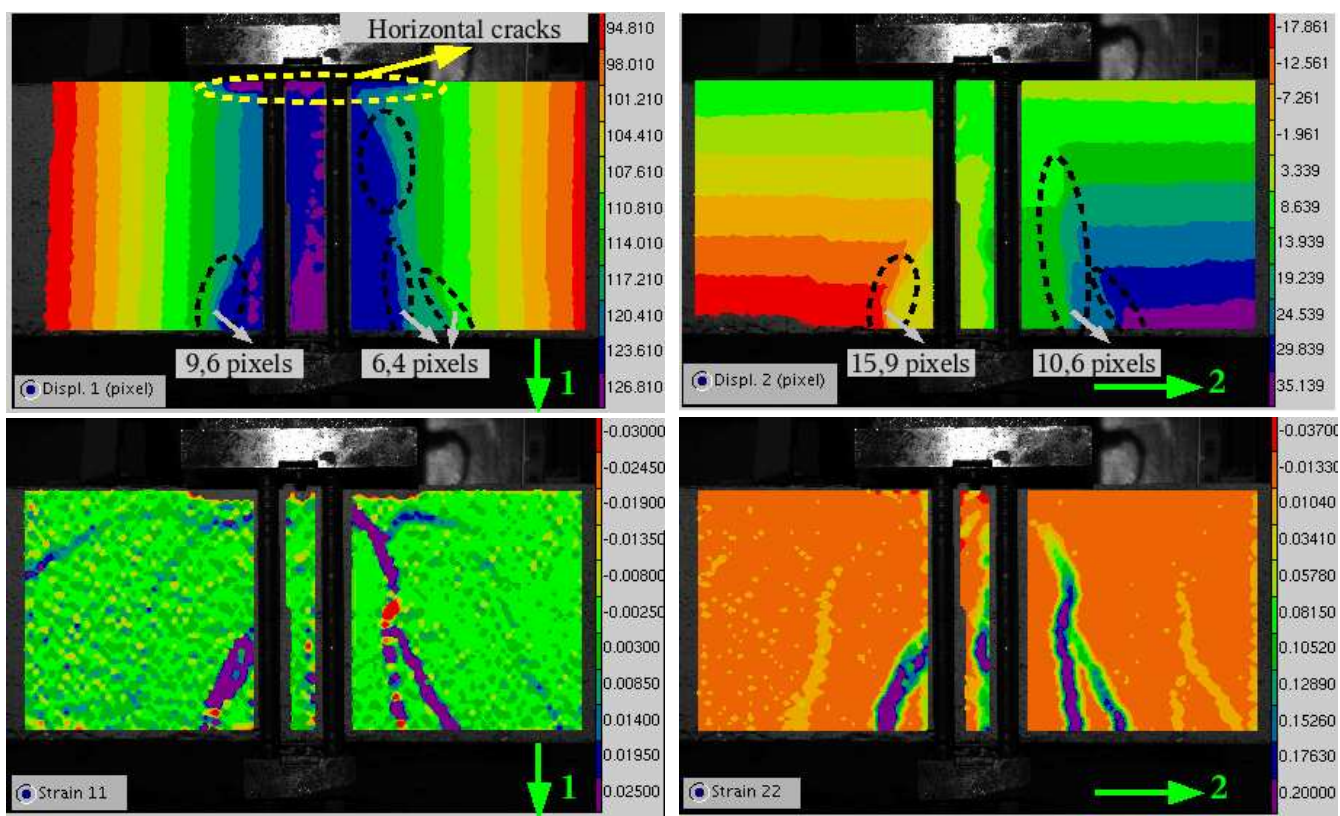

Fig. 10. Cracking pattern for R1: displacement field (top) and strain field (bottom).
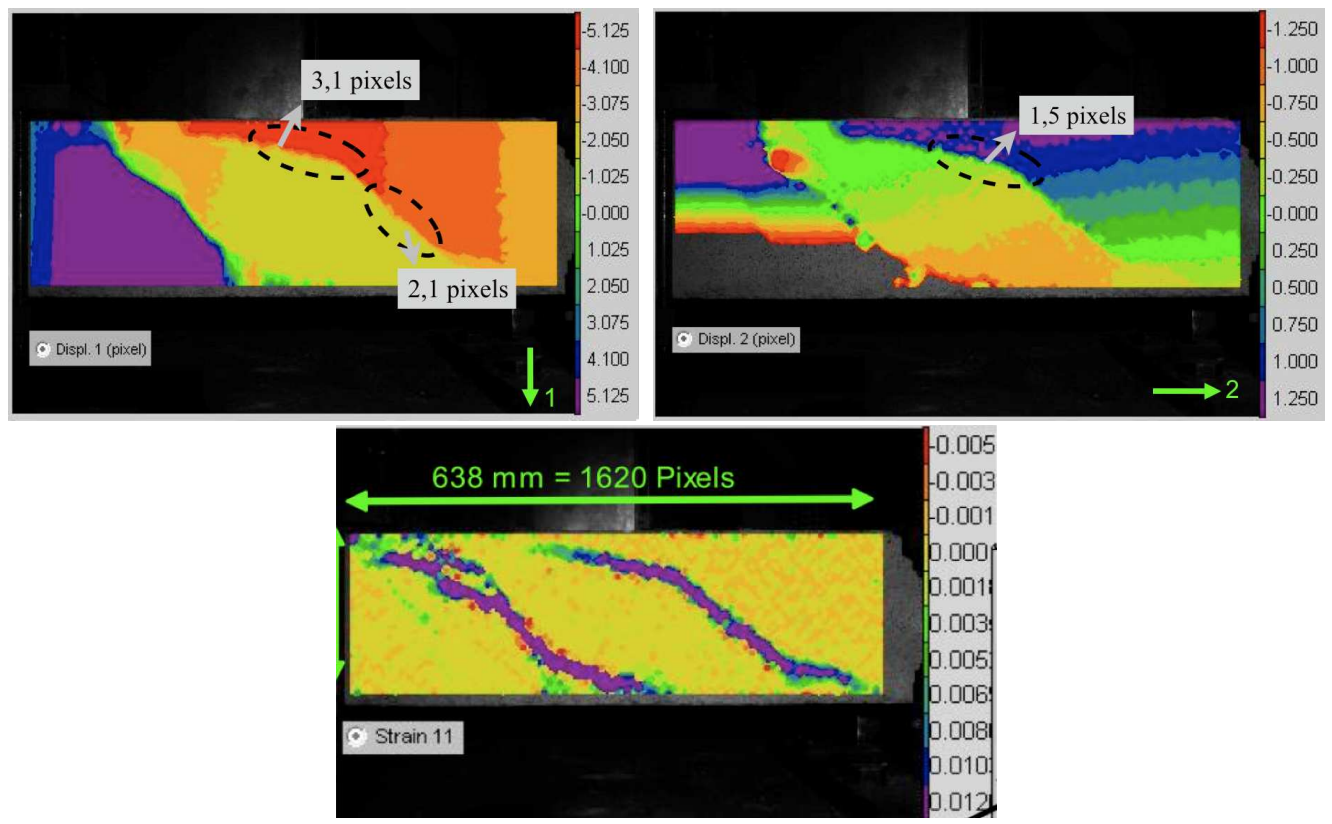

Fig. 11. Crack pattern for R2: displacement field (top) and strain field (bottom). 
Finally, results obtained for the three different beams are summarised in table 2. The advantage of the substructuring technique shows here its strong interest: different modes of failure have been investigated without testing three different whole structures.

\begin{tabular}{|c|c|c|c|c|c|}
\hline & Reinforcement & $\begin{array}{c}\text { Max. disp. } \\
(\mathrm{mm})\end{array}$ & $\begin{array}{c}\text { Max. } \\
\text { force }(\mathrm{kN})\end{array}$ & $\begin{array}{c}\text { Principal crack } \\
\text { orientation }\end{array}$ & $\begin{array}{c}\text { Max. crack } \\
\text { opening }(\mathrm{mm})\end{array}$ \\
\hline R1 & $4 \varnothing 10, \varnothing 6 / 15 \mathrm{~cm}$ & 23.8 & 55.2 & vertical & 2.3 \\
\hline R2 & $4 \varnothing 12, \varnothing 6 / 30 \mathrm{~cm}$ & 36.0 & 47.1 & inclined & 2.8 \\
\hline R3 & $4 \varnothing 8, \varnothing 6 / 15 \mathrm{~cm}$ & 38.3 & 30.2 & vertical & - \\
\hline
\end{tabular}

Table 2

General results.

Concerning the simulated substructure, damage occurs as expected. Anisotropic damage coupled with multifiber theory allows to distinguish the orientation of the crack in tension ( $D_{11}$, perpendicular to the beam axis) and in compression $\left(D_{22}\right.$, parallel to the beam axis). Figure 12 shows the components $D_{11}$ and $D_{22}$ of the damage tensor for the reinforcement $\mathrm{R} 1$ at the end of the static load application and at time $t=1.2 \mathrm{~s}$. The components are really similar for the other reinforcements and are not shown here. Taking into account the nonlinear behaviour is crucial for this dynamic loading, as the eigen frequencies of the whole structure are affected by the damage values. This effect can be seen on Figure 13 where the horizontal displacement of the upper left point is plotted versus time. Two numerical simulations are presented, considering a linear and a nonlinear behaviour for the simulated substructure, and the experimental nonlinear response of the tested beam. A spectral analysis of the two signals shows an important decrease of the first apparent eigen frequency, from $2.0 \mathrm{~Hz}$ (linear case) to $1.1 \mathrm{~Hz}$ (nonlinear case).

\section{Conclusion}

A study of a reinforced concrete structure submitted to an earthquake has been presented. Pseudo-dynamic tests are coupled with substructuring technique, a now classical approach accessible for many laboratories due to the simple experimental setup compared to the equivalent test on the whole structure. Note that representation of damping or any viscous and hysteretic effects are not accounted for in pseudodynamic tests and remains a major drawback of this method. The originality is to have consider an advanced damage model for concrete and a field measurement setup.

The anisotropic damage model is extended to cyclic and seismic loading con- 


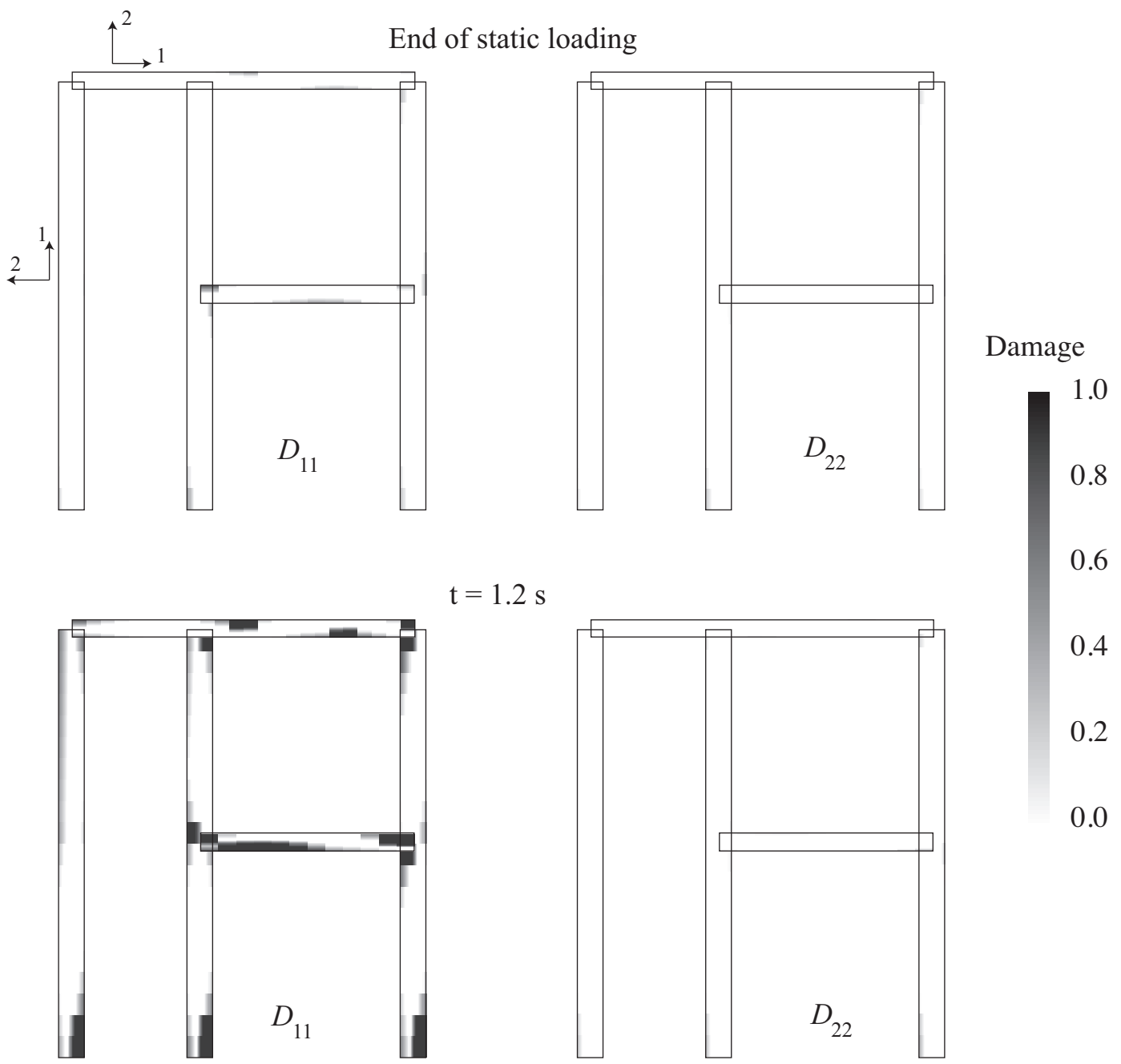

Fig. 12. Damage maps (in concrete) for the simulated structure: $D_{11}$ (left) and $D_{22}$ (right).

ditions by means of the concept of micro-cracks closure and damage deactivation. Only five parameters are introduced (including elastic parameters). The analysis shows the efficiency of such a nonlinear model used for the computed substructure when coupled with a simplified multifiber theory. This allows a rich description of the concrete, by taking into account the dissymmetry of the behaviour in tension and compression, the micro-cracks closure effect. The damage anisotropy model gives access to the crack orientation in the structure, even for the simplified 1D discretization.

The measurement setup uses digital image correlation technique. In that way, a full field information (displacement and strain fields) is obtained instead of a local one. A fine crack description is obtained during the loading, allowing the measure of the crack opening.

Results are shown for different typical beam reinforcements. Hardening of 


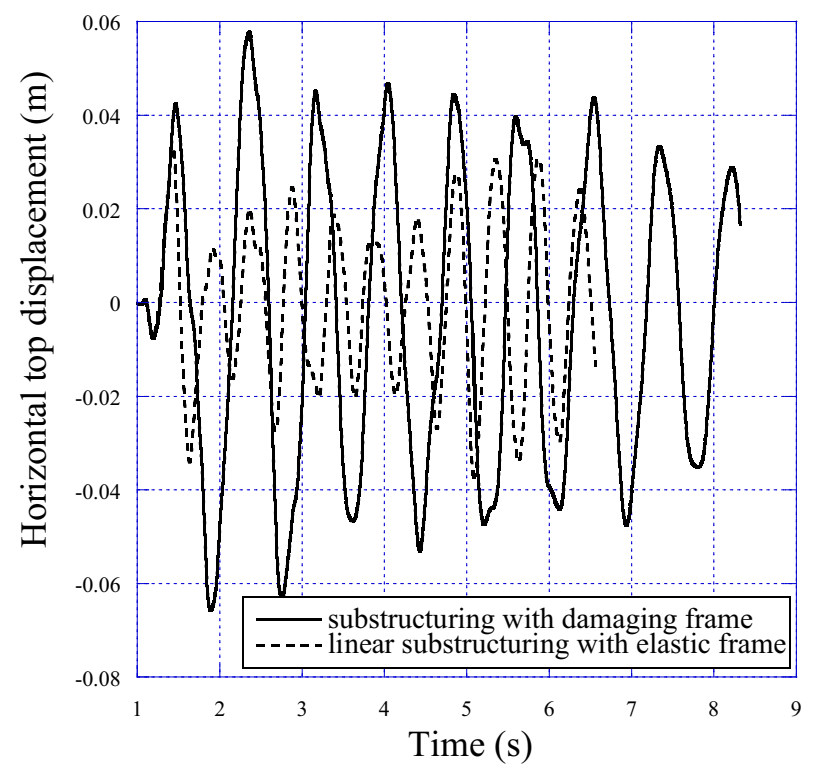

Fig. 13. Horizontal displacement of the upper left point of the whole structure: effect of nonlinearities (elastic frame vs damaging frame).

the steel bars, shear failure mode, tensile fracture of the longitudinal reinforcement have been observed. All the results corresponding to seismic failure modes have been obtained with a simple three point bend test, thanks to the pseudo-dynamics analysis with substructuring technique. Combining with digital image correlation and robust non linear computation, this approach gives a quite easy access to studies of complex failure modes of structures.

\section{Acknowledgements}

The authors would like to thank F.Hild for his kind advices and help for image correlations.

\section{References}

[1] AFNOR. Règles PS applicables aux bâtiments, dites Règles PS92. NF P 06-013 / DTU Règles PS92; 1995, pp. 516-536 (in french).

[2] Bazant ZP, Pan JY, Pijaudier-Cabot G. Softening in reinforced concrete beams and frames, ASCE J. of Struct. Engrg., 1987, Vol. 113, pp. 2333-2347.

[3] Buchet P, Pegon P. PsD testing with substructuring implementation and use. special publication 1994, 1.94.25 JRC ISPRA. 
[4] Chaboche JL. Development of continuum Damage Mechanics for elastic solids sustaining anisotropic and unilateral damage. Int. J. Damage Mechanics 1993, Vol. 2, pp. 311-329.

[5] Chang SY. Application of the momentum equation of motion to pseudodynamic testing. Phil. Trans. R. Soc. London 2001, Vol. 359, pp.1801-1827.

[6] Chung J, Yun CB, Kim AS, and Seo JW. Shaking table and pseudo dynamic tests for the evaluation of the seismic performance of base-isolated structure. Engng. Struct. 1999, Vol. 21, pp. 365-379.

[7] Combescure D. Modélisation des structures de génie civil sous chargement sismique à l'aide de Castem2000. CEA publication 2001; ref. SEMT/EMSI/RT/016008A, France, www-cast3m.cea.fr/ (in french).

[8] Combescure, D. and Pegon, P., $\alpha$-Operator splitting time integration technique for pseudodynamic testing error propagation analysis, Soil Dynamics and Earthquake Engineering, 1997, Vol. 16(7-8), pp. 427-443.

[9] Desmorat R, Gatuingt F, Ragueneau F. Nonlocal anisotropic damage model and related computational aspects for quasi-brittle materials. Engineering Fracture Mechanics, 2007, Vol 74(10), pp. 1539-1560.

[10] Haussman G., The CU-Boulder Fast Hybrid Test Desktop Platform, CU-NEES06-3, University of Colorado at Boulder, 2006.

[11] Dragon A, Halm D. Modélisation de l'endommagement par mésofissuration: comportement unilatéral et anisotropie induite. C. R. Acad. Sci. 1996, t. 322, Série IIb, p. 275-282 (in french).

[12] Gravouil A. Méthodes multiéchelles en temps et en espace avec décomposition de domaines pour la dynamique nonlinéaire des structures. Ph.D. thesis. LMTCachan(ENS Cachan/CNRS/Université Paris 6/UniverSud Paris), France; 2000 (in french).

[13] Guedes J, Pegon P, Pinto AV. A fiber/Timoshenko Beam Element in CASTEM 2000. JRC Special Publication 1994, Nr.I.94.31.

[14] Hamam, R., Hild, F., Roux, S. Stress intensity factor gauging by digital image correlation : Application in cyclic fatigue. Strain, 2007, Vol 43, pp. 181-192.

[15] Hilbert HM, Hughes TJR, Taylor RL. Improved numerical dissipation for time integration algorithms in structural dynamics. Earthquake Engineering and Structural Dynamics 1977, Vol. 5, pp. 283-292.

[16] Hild F. CORRELI ${ }^{L M T}$ : a software for displacement field measurements by digital image correlation. LMT-Cachan (ENS Cachan/CNRS/Universite Paris 6/UniverSud Paris), France 2002; Internal report N 254.

[17] Hild, F., Roux, S. Measuring stress intensity factors with a camera : integrated digital image correlation (I-DIC). Comptes-Rendus Mecanique, 2006, Vol 334(1), pp. 8-12. 
[18] Hild F, Roux S. Full-field measurements and identification in solid mechanics. Lecture notes, Vol. 1, ISBN 978-83-89687-13-5, KMM-NoE Network of Excellence; Warsaw 2007.

[19] Horiuchi T, Nakagawa M, Sugano M, Konno T. Development of a real-time hyprid experimental system with actuator delay compensation. Proceeding of 11th World Conference on Earthquake Engineering, 1996.

[20] Laborderie C. Phénomènes unilatéraux dans un matériau endommageable : modélisation et application à l'analyse de structures en béton. Ph.D. thesis. LMT-cachan (ENS Cachan/CNRS/Universite Paris 6/UniverSud Paris), France; 1991 (in french)

[21] Ladevèze P. On an anisotropic damage theory Proc. CNRS Int. Coll. 351 Villarsde-Lans, Failure criteria of structured media, Edited by J. P. Boehler. 1983, pp. 355-363.

[22] Lemaitre J, Desmorat R, Sauzay M. Anisotropic damage law of evolution, Eur. J. Mech., A/Solids 2000, Vol. 19, pp. 187-208.

[23] Lemaitre J, Desmorat R., Engineering damage mechanics: ductile, creep, fatigue and brittle failures. Springer; 2005.

[24] Mahin S, Shing PB. Pseudodynamic method for seismic testing, J. Struct. Engng. 1985, Vol. 111, pp. 1482-1503.

[25] Mazars J. Application de la mécanique de l'endommagement au comportement non linéaire et à la rupture du béton de structure. Thèse d'état. U. Paris 6; 1984 (in french).

[26] Mazars J., Kotronis P., Ragueneau F. and Casaux G., Using multifiber beams to account for shear and torsion: Applications to concrete structural elements, Computer Methods in Applied Mechanics in Engineering, 2006, Vol. 195(52), pp. 7264-7281.

[27] Nakashima, M., Akazawa, T. and Sakaguchi, O., Integration method capable of controlling experimental error growth in substructure pseudo dynamic test, AIJ J. of Struct. Constr. Engng., 1993, Vol. 454, pp. 61-71.

[28] Pegon P, Pinto AV. Pseudo-dynamic testing with substructuring at the ELSA laboratory. Engng. Struct. Dyn 2000, Vol. 29, pp. 905-925.

[29] Pegon P, Magonette G. Continuous PsD testing with non-linear substructuring: presentation of a stable parallel Inter-Field procedure. JRC special publication 2002, No. I.02.167.

[30] Proenca JM, Calado L. Development of pseudodynamic test method and evaluation of errors. Proceeding of 11th European Conference on Earthquake Engineering. 1998 Balkema; Roterdam, ISBN 9054109823.

[31] Shing, B., Vannan, M.T. and Cater, E., Implicit time integration for pseudodynamic tests, Earthquake Eng. Struct. Dyn., 1991, Vol. 20, pp. 551576. 
[32] Simo J, Hughes T. Computational inelasticity. Springer; 1998.

[33] Sollogoub P, Combescure D, Queval JC, Chaudat Th. In plane behaviour of several $1 / 3$ rd scaled RC bearing walls. Testing and interpretation using non linear modelling. 12th World Conference on Earthquake Engineering, Auckland, New Zealand. January 2000.

[34] Souid A. Essais PSeudo-Dynamiques sur structures : schémas d'intégration temporelle et analyse paramétrique. Rapport de stage DEA MaiSE. LMTCachan (ENS Cachan/CNRS/Universite Paris 6/UniverSud Paris), France; 2003 (in french).

[35] Souid A, Delaplace A, Ragueneau F, Desmorat R. Pseudodynamic tests and substructuring of damageable structures. Sixth European Conference on Structural Dynamics, Paris, France. 2005.

[36] Spacone E, Filippou FC, Taucer FF. Fiber Beam-Column Model for Nonlinear Analysis of R/C Frames. I: Formulation. Earthquake Engineering and Structural Dynamics 1996, Vol. 25, pp. 711-725.

[37] M.A. Sutton, S.R. McNeill, J.D. Helm, Y.J. Chao, Advances in TwoDimensional and Three-Dimensional Computer Vision, in: Photomechanics, Springer, 2000, pp. 323-372.

[38] Takanashi K, Nakashima M. Japonese activities online testing, Engng. Mech. 1987, Vol. 113, pp. 1014-1032.

[39] Williams DM. Numerical modelling of real-time sub-structure testing, Ph.D. thesis. Keble college, Oxford; 2000.

[40] Williams MS, Blakeborough A. Laboratory testing of structures under dynamic loads: an introductory review. Phil. Trans. R. Soc. Lond. 2001, pp. 1651-1669.

[41] Zienkiewicz OC, Taylor RL. The finite element method. Vol. I, II and III, 5th. Ed. Butterworth Heinemann, Oxford; 2000. 